\title{
Distributed forward stimulated Brillouin scattering measurement using broadband BOTDR
}

Simon Zaslawski, Zhisheng Yang, Sheng Wang, Luc Thévenaz

Simon Zaslawski, Zhisheng Yang, Sheng Wang, Luc Thévenaz, "Distributed forward stimulated Brillouin scattering measurement using broadband BOTDR," Proc. SPIE 11199, Seventh European Workshop on Optical Fibre Sensors, 1119923 (28 August 2019); doi: 10.1117/12.2540011

Event: Seventh European Workshop on Optical Fibre Sensors, 2019, Limassol, Cyprus 


\title{
Distributed forward stimulated Brillouin scattering measurement using broadband BOTDR
}

\author{
Simon Zaslawski ${ }^{\mathrm{a}}$, Zhisheng Yang*a, Sheng Wang ${ }^{\mathrm{a}, \mathrm{b}}$, Luc Thévenaz ${ }^{\mathrm{a}}$ \\ ${ }^{a}$ EPFL Swiss Federal Institute of Technology, Group for Fibre Optics, SCI-STI-LT Station 11, \\ 1015 Lausanne, Switzerland; 'Beijing University of Posts and Telecommunications, 100876 Beijing, \\ China \\ *Email: zhisheng.yang@epfl.ch
}

\begin{abstract}
Brillouin optical time-domain reflectometry is used to perform distributed forward stimulated Brillouin scattering (FSBS) measurements. This configuration suppresses the need for an additional frequency scanning to evaluate the local Brillouin peak gain when probing the FSBS resonance. The use of a broad pass-band filter makes the system insensitive to moderate temperature or strain fluctuations, but enables to accurately retrieve any change in intensity due to FSBS.
\end{abstract}

Keywords: Optical fibre sensor, stimulated Brillouin scattering

\section{INTRODUCTION}

Recently, guided acoustic waves Brillouin scattering (GAWBS) or forward stimulated Brillouin scattering ${ }^{1}$ (FSBS) gained a substantial interest within the sensing community as it might broaden the range of parameters to which distributed fibre sensors are sensitive. FSBS is a flavour of Brillouin scattering that involves acoustic modes resonating in the fibre cross-section ${ }^{2}$. Although the physical effects leading to FSBS generation are similar to those involved in backward stimulated Brillouin scattering (SBS), its exploitation for sensing applications requires a major update of the sensor design. Since transverse acoustic waves are sensitive to the fibre outer interface conditions, FSBS is highly dependent on the cladding surroundings, notably on the fibre coating ${ }^{3,4}$. Furthermore, FSBS is a forward scattering process that will accumulate as light propagates down the fibre, making a distributed measurement more challenging. Techniques reported so far to circumvent this difficulty involve either measuring the Rayleigh backscattering of two optical tones ${ }^{5}$ or decoupling of the FSBS activation from the interrogating scheme ${ }^{6}$. One of the limitations experienced so far is the need to perform an additional time-consuming scanning for each probed acoustic frequency.

In this work, we propose to measure distributed FSBS based on Brillouin optical time-domain reflectometry (BOTDR), associated to a broadband electrical filter. This filter is designed to entirely cover the classical Brillouin gain spectrum (BGS), such that the system will only respond to intensity changes in the backscattered signal. This suppresses the classical Brillouin gain frequency scanning, only keeping the scanning of the FSBS resonance. The significant time saved by skipping one scanning can be invested to perform more averages, leading to an increase in signal-to-noise ratio for identical measurement times. Distributed FSBS measurements were performed over $\sim 650 \mathrm{~m}$ of sensing fibre with a spatial resolution of $8 \mathrm{~m}$, which represents an increase by a factor $\sim 2$ compared to previous results ${ }^{6}$.

\section{EXPERIMENTAL IMPLEMENTATION}

The implementation is fairly identical to a classical Brillouin optical time-domain reflectometer (BOTDR), as illustrated in Fig. 1. It is worth to mention that, unlike most configurations, the RF filter is not here selected to finely sample the Brillouin gain spectrum (BGS), but must collect all its energy at once. For FSBS activation, a strong pulse - in red - is generated from a laser operating at $\sim 1530 \mathrm{~nm}$. The modulating frequency $f_{\mathrm{FSBS}}$ lies within the FSBS resonance of a specific transverse acoustic mode. The reading pulse -in blue- is emitted at $\sim 1550 \mathrm{~nm}$ and immediately follows the activating pulse. A tunable filter prevents the strong backscattered signal from the activating pulse from reaching the photodetector and jamming the measurement. The electro-optic modulator (EOM) is driven in carrier-suppressed mode at a fixed arbitrary frequency $f_{\mathrm{LO}}$ to generate a local oscillator (LO) signal. The backscattered signal from the reading pulse is then measured via heterodyne detection using a balanced photo-detector (BPD). The use of a band-pass filter (BPF) is compulsory to prevent the strong LO signal from reaching the envelope detector. The polarization scrambler

Seventh European Workshop on Optical Fibre Sensors, edited by Kyriacos Kalli, Gilberto Brambilla, Sinead O'Keeffe Proc. of SPIE Vol. 11199, 1119923 · C 2019 SPIE · CCC code: 0277-786X/19/\$21 · doi: 10.1117/12.2540011 
fulfils two purposes: 1) it enables to minimise the contribution from torsional-radial acoustic modes ${ }^{2}$ that may overlap with the targeted purely radial modes; 2) it also provides a mean of averaging the polarization-induced intensity variations in the coherent detection process.

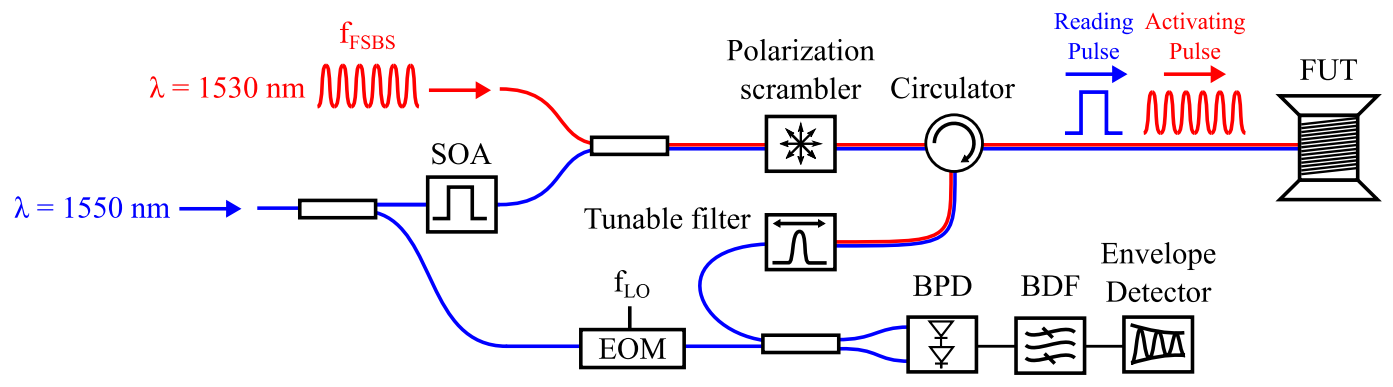

Figure 1. Experimental setup for distributed FSBS measurements. The activation pulse follows the red channel while the reading blue channel shares similarities with a standard BOTDR setup. SOA: semiconductor optical amplifier, FUT: fibre under test, EOM: electro-optic modulator, BPD: balanced photo-detector, BPF: band-pass filter.

FSBS manifests on the reading pulse as a cumulative phase-modulation process, leading to the generation of harmonics that evolve with distance following Bessel functions distributions. By monitoring the temporal evolution of several of these harmonics, the local phase modulation depth can be readily retrieved ${ }^{6}$. Fig. 2 presents a synthetic view of all frequencies in our system, and the multiple combinations eventually put some restrictions on the choice of the BPF.

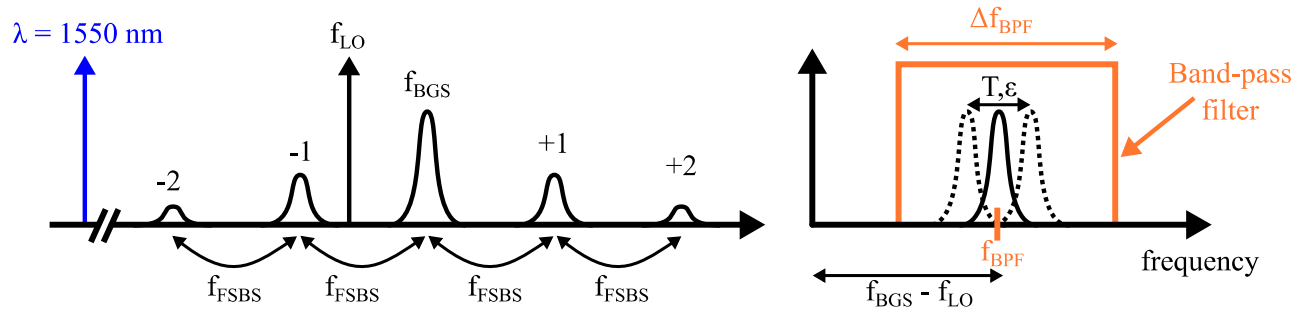

Figure 2. Illustration of the various frequencies relevant to the choice of the band-pass filter used for distributed FSBS measurements. In this example, the local oscillator is positioned to collect the fundamental spectrum (BGS) while rejecting the higher order harmonics.

The passband $\Delta f_{\mathrm{BPF}}$ must be broad enough to entirely cover the full Brillouin gain spectrum (BGS), including variations of the BGS frequency with position, for instance due to strain or temperature. This makes this measurement both temperature and strain insensitive, provided that the BGS variations around its mean value remain within the filter passband.

\section{RESULTS}

The $\Delta f_{\mathrm{BPF}}=100 \mathrm{MHz}$ band-pass filter (BPF) was centered at $f_{\mathrm{BPF}}=550 \mathrm{MHz}$ and enabled to perform distributed FSBS measurements with a spatial resolution of $8 \mathrm{~m}$. The selected acoustic mode was activated by a $400 \mathrm{~ns}$ long pulse, modulated in intensity around $f_{\mathrm{FSBS}}=370 \mathrm{MHz}$.

\subsection{Broadband BOTDR}

The method described in the previous section relies entirely on the ability of the BPF to collect the entire spontaneous Brillouin backscattering from the reading pulse. This was checked by turning off the activating pulse and by recording the system response as the local oscillator frequency $f_{\mathrm{LO}}$ was swept from $10 \mathrm{GHz}$ to $10.6 \mathrm{GHz}$. The results are depicted in Fig. 3, in which the strong asymmetry in the recorded spectrum is a direct consequence of using an asymmetric BPF, built by cascading a low-pass filter and a high-pass filter. This prior measurement enables to spot a suitable operating range, since the obtained spectrum shows a flat region between $f_{\mathrm{LO}}=10.31 \mathrm{GHz}$ and $f_{\mathrm{LO}}=10.34 \mathrm{GHz}$, as illustrated in the inset of Figure 3. Since the acquired signal is constant over this interval, the BGS is in all cases entirely covered. This frequency range also determines the maximum frequency range over which the BGS may fluctuate without significantly impairing the measurement. After a careful examination, it turns out that the ideal LO frequency to measure the BGS is $10.322 \mathrm{GHz}$. 


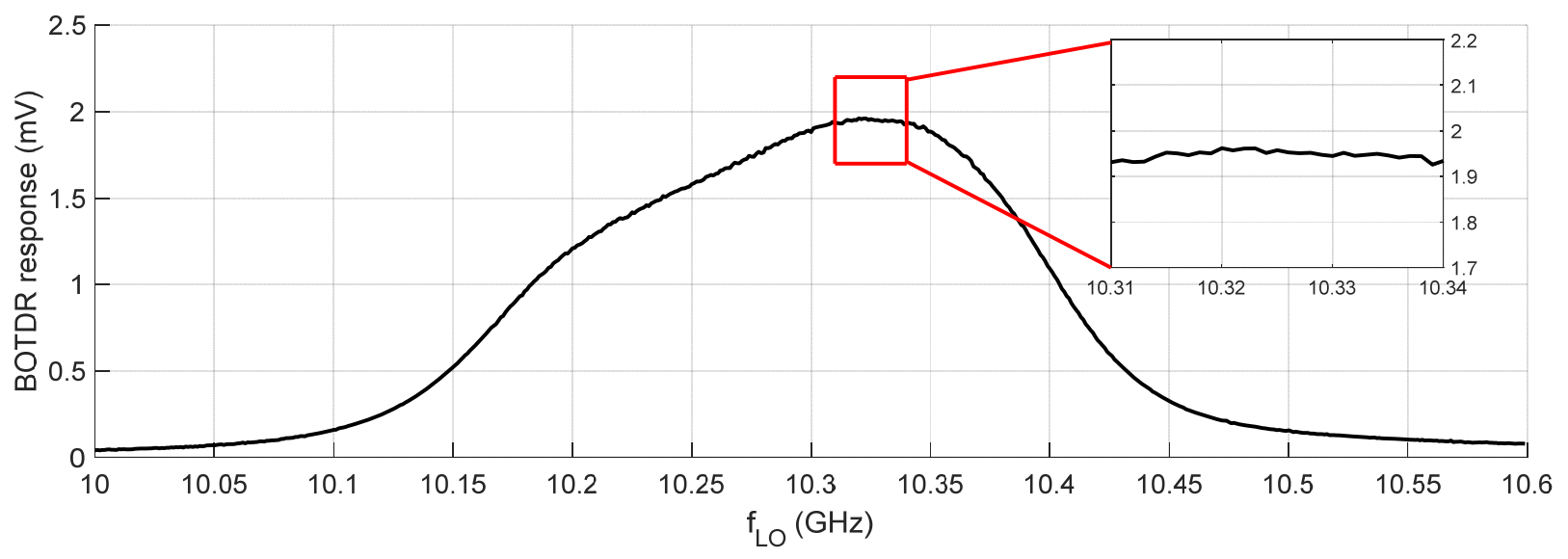

Figure 3. Response of the broadband BOTDR setup in the absence of activating pulse.

\subsection{Distributed FSBS measurements}

Due to its transverse nature, FSBS lifetime is very dependent on the acoustic properties of the fibre surrounding, in particular the impedance mismatch at the cladding-coating interface ${ }^{2-6}$. FSBS turns out to be poorly sustained in standard single-mode fibres, as the thick acrylate coating - usually $62.5 \mu \mathrm{m}$ thick - acts as a strong acoustic damper. The fibre tested in this work is therefore chemically stripped of its coating over some $30 \mathrm{~m}$. The sensor response is significantly enhanced, as air and fused silica present a strong impedance mismatch. This section of the fibre is later immersed in ethanol to validate the sensing capabilities of the setup. The local oscillator frequency $f_{\text {Lo }}$ is set to $10.322 \mathrm{GHz}$, $9.952 \mathrm{GHz}$ and $9.582 \mathrm{GHz}$ to capture the intensity of the BGS $\left(\mathrm{I}_{0}\right)$, the first sideband $\left(\mathrm{I}_{1}\right)$ and the second sideband $\left(\mathrm{I}_{2}\right)$, respectively (see Fig. 2). In each case, the FSBS resonance is probed by sweeping the exciting pulse modulation frequency between $364 \mathrm{MHz}$ and $377 \mathrm{MHz}$ with a $0.1 \mathrm{MHz}$ scanning step. The proper combination of $I_{0}, I_{1}$, and $I_{2}$ enables to compute ${ }^{6}$

$$
\zeta(\mathrm{z})=\frac{2 \sqrt{\mathrm{I}_{1}}}{\sqrt{\mathrm{I}_{0}}+\sqrt{\mathrm{I}_{2}}}=\frac{2 \pi}{\lambda} \int_{0}^{\mathrm{z}} \Delta \mathrm{n}\left(\mathrm{z}^{\prime}\right) \mathrm{dz} \mathrm{z}^{\prime}
$$

where $\zeta(z)$ is the cumulated phase modulation depth as a function of distance $z, \lambda$ is the reading pulse wavelength and $\Delta n\left(z^{\prime}\right)$ is the local amplitude of refractive index change induced by the FSBS vibration in the core region. Fig. 4(a) and (b) show the measured $\zeta(z)$ and $\Delta n\left(z^{\prime}\right)$ at FSBS resonance $\left(f_{\mathrm{FSBS}}=370 \mathrm{MHz}\right)$, respectively, where $\Delta n\left(z^{\prime}\right)$ is obtained from $\zeta(z)$ by numerical differentiation. Fig. 4(c) shows the measured FSBS resonance in the uncoated section of the test fibre. Due to its lower impedance mismatch, the response is much broader for ethanol than for air. The full width at halfmaximum (FWHM) measured are $F W H M_{\text {air }}=2.15 \mathrm{MHz}$ and $F W H M_{\text {eth }}=3.62 \mathrm{MHz}$ for air and ethanol, respectively. These values cannot be used for the determination of the acoustic impedance, as the curves shown in Fig. 4(c) correspond to the convolution between the intrinsic acoustic resonance and the activating pulse spectrum. Following the same methodology described in a previous work ${ }^{6}$, the intrinsic linewidth are $\delta \mathrm{f}_{\text {eth }}=2.32 \mathrm{MHz}$ for ethanol and $\delta \mathrm{f}_{\text {air }}=0.1 \mathrm{MHz}$ for air. The acoustic impedance of ethanol $Z_{\text {eth }}$ is eventually given by ${ }^{6}$

$$
\mathrm{Z}_{\text {eth }}=\mathrm{Z}_{\mathrm{f}} \frac{\mathrm{k}-1}{\mathrm{k}+1} \quad \text { with } \quad \mathrm{k}=\exp \left(\pi \frac{\delta \mathrm{f}_{\text {eth }}-\delta \mathrm{f}_{\text {air }}}{\Delta \mathrm{f}_{\mathrm{m}}}\right)
$$

where $Z_{\mathrm{f}}=13.1 \times 10^{6} \mathrm{kgm}^{-2} \mathrm{~s}^{-1}$ is the tabulated acoustic impedance of fused silica ${ }^{7}$, and $\Delta f_{\mathrm{m}}=48 \mathrm{MHz}$ is the average frequency interval between the selected FSBS mode and the two adjacent ones. We found $Z_{\text {eth }}=0.95 \mathrm{kgm}^{-2} \mathrm{~s}^{-1}$, in good agreement with the tabulated value $\mathrm{e}^{7} 0.93 \mathrm{kgm}^{-2} \mathrm{~s}^{-1}$. 

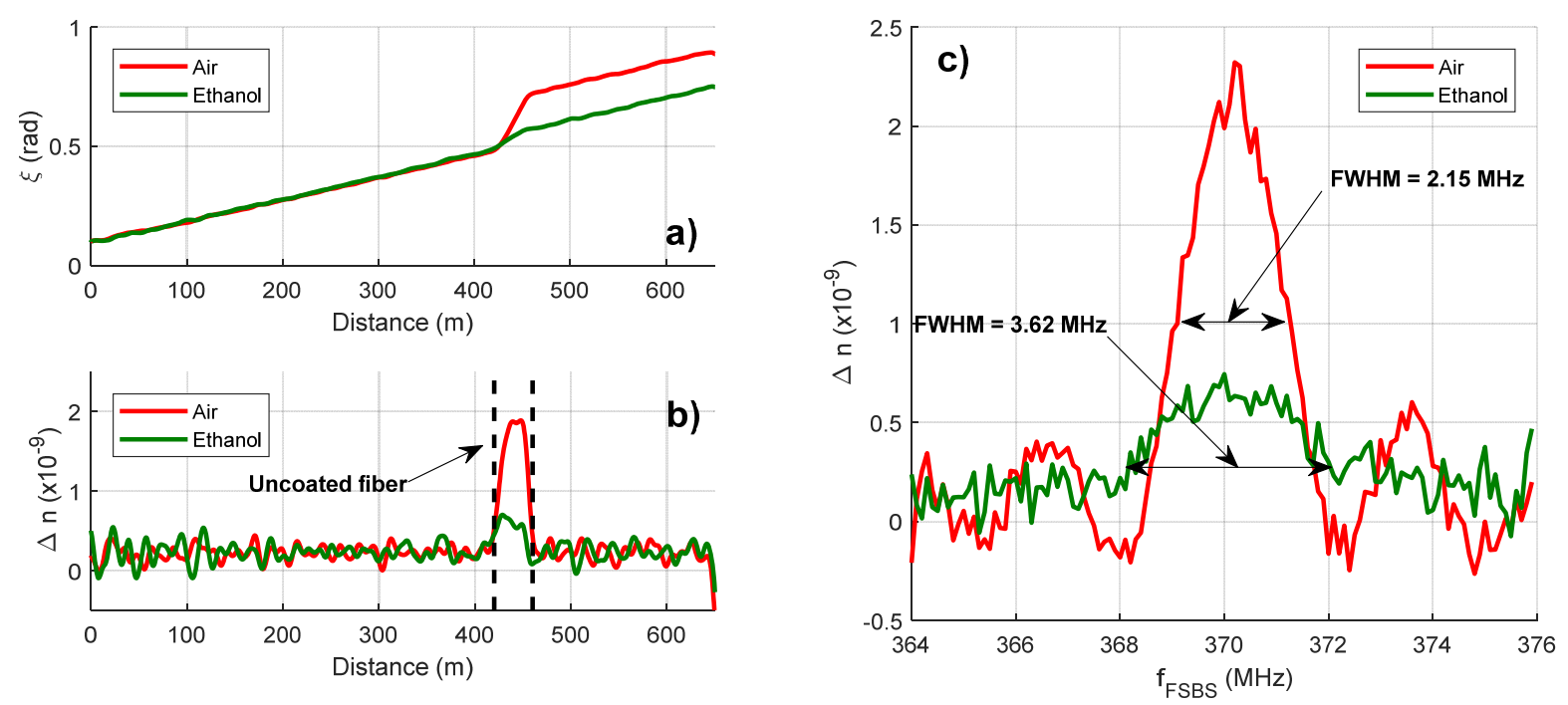

Figure 4.a) Phase cumulated by the reading pulse during propagation at resonance ( $\left.f_{\mathrm{FSBS}}=370 \mathrm{MHz}\right)$. b) Local refractive index change obtained from b) via numerical differentiation. c) Retrieved FSBS spectrum in the stripped fibre (450 m). The red curves correspond to the situation were the uncoated fibre was exposed to air, while the green curves were measured while bathing the uncoated fibre in ethanol.

\section{CONCLUSION}

Distributed FSBS measurements have been achieved with $8 \mathrm{~m}$ of spatial resolution over $650 \mathrm{~m}$ of standard single-mode fibre. The use of a $100 \mathrm{MHz}$ band-pass filter makes the system insensitive to slight deviations of the BGS central frequency while capturing efficiently the intensity variations due to FSBS. Ultimately, the system was found to be exclusively limited by signal-to-noise ratio (SNR), which may be enhanced in several ways besides increasing the averaging. The use of fibres with thin polyimide coating can potentially further improve the performances of the system due to their more suitable acoustic property. This includes, first, a stronger response compared to standard acrylate coating due to lower damping, and second, a shift of the dominant FSBS modes to higher frequencies. This will give more flexibility in the filter choice with a lower central frequency, hence switch to a photodetector with improved noise performances.

The authors acknowledge the support from the Swiss National Science Foundation (project number: 178895).

\section{REFERENCES}

[1] Shelby, R. M., Levenson, M. D. and Bayer, P. W., "Guided acoustic-wave Brillouin scattering," Phys. Rev. B 31, 5244-5252 (1985). displays," SID Int. Symp. Digest Tech. Papers 32(1), 934-937 (2001).

[2] Antman, Y., Clain, A. and London, Y., "Optomechanical sensing of liquids outside standard fibres using forward stimulated Brillouin scattering," Optica 3(5), 510-516 (2016).

[3] Chow, D. M. and Thévenaz, L., "Forward Brillouin scattering acoustic impedance sensor using thin polyimidecoated fibre," Opt. Lett. 43(21), 5467-5470 (2018).

[4] Diamandi, H. H., London, Y., Bashan, G. and Zadok A., "Distributed Opto-Mechanical Sensing Outside Polyimide-Coated Fiber," 26th International Conference on Optical Fiber Sensors, FB1 (2018).

[5] Bashan, G., Diamandi, H. H., London, Y., Preter, E. and Zadok, A., "Optomechanical time-domain reflectometry," Nat. commun. 9(2991), (2018)

[6] Chow, D. M., Yang, Z. and Thévenaz, L., "Distributed Forward Brillouin sensor based on local light phase recovery," Nat. commun. 9(2990), (2018)

[7] NDT Education Resource Center., "Material Property Tables,"

$<$ https://www.nde-ed.org/GeneralResources/MaterialProperties/UT/ut_matlprop_index.htm> 